\title{
An efficient algebraic multigrid method for second order elliptic equations on polygonal domains
}

\author{
Ming $\operatorname{Li}^{1}$ \\ ${ }^{1}$ Honghe University
}

May 14, 2021

\begin{abstract}
Based on a coarsening strategy of adjacency matrix, a new algebraic prolongation operator is developed for standard V-cycle multigrid method to accelerate the whole process. An efficient algebraic multigrid (EAMG) method is proposed for solving large-scale linear systems, arising from finite element (FE) discretization of second order elliptic boundary value problems. Numerical experiments on polygonal domains are conducted to demonstrate the EAMG computation is more efficient than standard method.
\end{abstract}

\section{Hosted file}

MMA-EAMG-20210507.pdf available at https://authorea.com/users/413870/articles/522040-anefficient-algebraic-multigrid-method-for-second-order-elliptic-equations-on-polygonaldomains

\section{Hosted file}

MMA-EAMG-20210507.tex available at https://authorea.com/users/413870/articles/522040-anefficient-algebraic-multigrid-method-for-second-order-elliptic-equations-on-polygonaldomains 
figures/graph-matrix/graph-matrix-eps-converted-to.pdf 
figures/coarsening-set/coarsening-set-eps-converted-to.pdf 
figures/SMesh-a4/SMesh-a4-eps-converted-to.pdf 
figures/SMesh-b4/SMesh-b4-eps-converted-to.pdf 
figures/SMesh-c3/SMesh-c3-eps-converted-to.pdf 
figures/L-unstructured2/L-unstructured2-eps-converted-to.pdf 
figures/U-unstructured1/U-unstructured1-eps-converted-to.pdf 